\title{
Fluid-structure interaction: analysis of a 3-D compressible model
}

by

\author{
Fabien FLORI ${ }^{1}$, Pierre ORENGA ${ }^{2}$ \\ URA CNRS 2053, University of Corsica, Grossetti, B.P. 52, 20250 Corte, France \\ Manuscript received 6 January 2000
}

\begin{abstract}
In this paper, we present an existence result of weak solutions for a three-dimensional problem of fluid-plate interaction in which we take into account the non linearity of the continuity equation. This non linearity does not allow, as is usually the case, to neglect the variations of the domain which leads us to study a problem defined on a time dependent domain.

(C) 2000 L'Association Publications de l'Institut Henri Poincaré. Published by Elsevier B.V. All rights reserved AMS classification: $35 \mathrm{Q} 30,73 \mathrm{C} 02,73 \mathrm{~K} 10,76 \mathrm{~N} 10$
\end{abstract}

Key words: Fluid and structure interaction, Classical linear elasticity, Plates, Compressible fluids

RÉSUMÉ. - Dans ce papier, nous présentons un résultat d'existence de solutions faibles pour un problème de couplage fluide-plaque tridimensionnel dans lequel nous prenons en compte la non linéarité de l'équation de continuité. Cette non linéarité ne nous permet pas, comme c'est généralement le cas, de faire l'hypothèse de petites perturbations et de négliger les variations du domaine ce qui nous conduit à étudier un problème défini sur un domaine dépendant du temps.

(C) 2000 L'Association Publications de l'Institut Henri Poincaré· Published by Elsevier B·V· All rights reserved

\footnotetext{
${ }^{1}$ E-mail: flori@univ-corse.fr.

${ }^{2}$ E-mail: orenga@univ-corse.fr.
} 


\section{INTRODUCTION}

We study a three-dimensional fluid-structure interaction problem in which the structure is a plate occupying a part of the fluid domain boundary. This problem has been already analyzed in a previous study [4] in a two dimensional situation. This first work already pointed out an important difficulty. Indeed, under conditions of weak disturbances, it is classically assumed that the fluid occupies a fixed domain $\Omega$. Nevertheless, in the more general case, when we consider the non linearity of the continuity equation, this assumption leads to some difficulties. Principally, we are unable to obtain a priori estimates and to show that the problem is well posed. In order to avoid this difficulty, we must take into account the displacements of the structure in the geometry of the fluid.

We set $\left.Q_{p}=\Omega_{p} \times\right] 0, T\left[\right.$ where $\Omega_{p}$ is an open subset of $\mathbb{R}^{2}$ which physically represents the plate at rest, $\left.\Sigma_{a}=\Gamma_{a} \times\right] 0, T$ [ where $\Gamma_{a}$ is a part of the fluid boundary assumed to be fixed, $\Sigma_{b}=\bigcup_{t \in] 0, T[} \Gamma_{b}(t) \times\{t\}$ where $\Gamma_{b}(t)$ is the deformation of the plate at time $t . \Omega_{p}$ and $\Gamma_{a}$ are assumed to be sufficiently smooth. We define in $\mathbb{R}^{4}, Q=\bigcup_{t \in] 0, T[} \Omega_{t} \times\{t\}$ with $\left.\Omega_{t}=\bigcup_{x \in \Omega_{p}}\right] u(x, t), 1[$ and where $u$ is the motion within the structure. We set $\Sigma$ as the lateral boundary of $Q$. We define $\Omega_{0}$ (respectively $\Omega_{T}$ ) as being the interior in $\mathbb{R}^{3}$ of the intersection of $\bar{Q}$ with $t=0$ (respestively $t=T)$. The section $\Omega_{s}=Q \cap\{t=s\}$ is continuous with respect to $s$ and never empty, if $u$ is a continuous function of $x$ and $t$. $\Omega_{s}$ represents the domain occupied by the fluid at $t=s$.

We note $v, p$ and $\rho$, the velocity, the pressure and the density of the fluid. We consider the state equation $p=a \rho^{\gamma}$ with $a$ and $\gamma$ chosen in $\mathbb{R}_{+}^{*}$. Afterwards we set $\gamma=1$ but the results are always true for $\gamma>1$. Moreover, $\bar{\rho}$ is the reference average value of density and $\mu$ and $\xi$ are the so-called Lamé viscosity coefficients. The problem associated with the fluid is the following

$$
(\mathcal{F}) \begin{cases}\bar{\rho} \frac{\partial v}{\partial t}-(\mu+\xi) \Delta v-\xi \nabla \operatorname{div} v+a \nabla \rho^{\gamma}=0, & \text { in } Q, \\ \frac{\partial \rho}{\partial t}+\operatorname{div}(v \rho)=0, \rho \geqslant 0, & \text { in } Q, \\ \operatorname{curl} v \wedge n=0, & \text { on } \Sigma, \\ v \cdot n_{a}=0, & \text { on } \Sigma_{a}, \\ v(t=0)=v_{0}(x), \rho(t=0)=\rho_{0}(x) \geqslant 0, & \text { in } \Omega_{0},\end{cases}
$$


The motion of the plate is governed by the biharmonic operator. This model can be improved with the introduction of an inertia term $-\Delta \frac{\partial^{2} u}{\partial t^{2}}$. This term, generally neglected, is important because it permits to obtain the continuity of $u$ with respect to $x$ and $t$, that is to say $u \in C^{0}\left(\bar{Q}_{p}\right)$, which allows to affirm that $\Omega_{t}$ is never empty. We note $D$ the rigidity of the plate, $m$ the surfacic mass, $I$ the inertia moment and $\bar{\sigma}$ the force applied to the plate due to the stress tensor of the fluid. The problem associated with the plate is

$$
(\mathcal{S}) \begin{cases}m \frac{\partial^{2} u}{\partial t^{2}}-m I \Delta \frac{\partial^{2} u}{\partial t^{2}}+D \Delta^{2} u=f+\bar{\sigma}, & \text { in } Q_{p}, \\ u=\nabla u \cdot n_{p}=0, & \text { on } \partial Q_{p}, \\ u(t=0)=u_{0}(x), & \text { in } \Omega_{p}, \\ \frac{\partial u}{\partial t}(t=0)=u_{1}(x), & \text { in } \Omega_{p} .\end{cases}
$$

The unitary exterior normal $n_{b}=\left(\cos \left(\omega_{1}\right), \cos \left(\omega_{2}\right), \cos \left(\omega_{3}\right)\right)$ of $\Omega_{t}$ on $\Gamma_{b}$ is defined by

$$
\begin{aligned}
\cos \left(\omega_{1}\right) & =\frac{\partial u / \partial x_{1}}{\sqrt{1+(\mathrm{d} u / \mathrm{d} \bar{x})^{2}}}, \quad \cos \left(\omega_{2}\right)=\frac{\partial u / \partial x_{2}}{\sqrt{1+(\mathrm{d} u / \mathrm{d} \bar{x})^{2}}}, \\
\cos \left(\omega_{3}\right) & =\frac{-1}{\sqrt{1+(\mathrm{d} u / \mathrm{d} \bar{x})^{2}}},
\end{aligned}
$$

with

$$
\left(\frac{\mathrm{d} u}{\mathrm{~d} \bar{x}}\right)^{2}=\left(\frac{\partial u}{\partial x_{1}}\right)^{2}+\left(\frac{\partial u}{\partial x_{2}}\right)^{2}
$$

So, it can be written

$$
n_{b}=-n \cdot \cos \left(\omega_{3}\right)
$$

with

$$
n=\left(\frac{\partial u}{\partial x_{1}}, \frac{\partial u}{\partial x_{2}},-1\right) .
$$

We suppose that the normal velocity $v \cdot n_{b}$ at the plate-fluid interface is equal to the projection of the displacement velocity on the normal direction. So, we obtain the following coupling condition

$$
\text { (C) }\left\{v \cdot n_{b}=\frac{\partial u}{\partial t} \cos \left(\omega_{3}\right), \text { on } \Sigma_{b}\right.
$$


which can be noted

$$
v . n=-\frac{\partial u}{\partial t}, \quad \text { on } \Sigma_{b} .
$$

Moreover, as $\mathrm{d} \Gamma_{b}=\sqrt{(\mathrm{d} \bar{x})^{2}+(\mathrm{d} u)^{2}}$, then $\forall \Theta\left(x_{1}, x_{2}, u\right) \in L^{1}\left(\Gamma_{b}\right)$,

$$
\int_{\Gamma_{b}} \Theta \mathrm{d} \Gamma_{b}=\int_{\Omega_{p}} \Theta \sqrt{1+\left(\frac{\mathrm{d} u}{\mathrm{~d} \bar{x}}\right)^{2}} \mathrm{~d} \bar{x}=-\int_{\Omega_{p}} \Theta \frac{1}{\cos \left(\omega_{3}\right)} \mathrm{d} \bar{x}
$$

and in particular, for all $\sigma$ smooth enough, if $\Theta=\sigma v \cdot n_{b}$, we have

$$
\int_{\Gamma_{b}} \sigma v \cdot n_{b} \mathrm{~d} \Gamma_{b}=-\int_{\Omega_{p}} \sigma \frac{\partial u}{\partial t} \mathrm{~d} \bar{x} .
$$

Lastly, we assume that the force applied by the fluid to the plate can be approximated by $[2,3]$

$$
\bar{\sigma}\left(x_{1}, x_{2}, u\right)=-a \rho^{\gamma}+(\mu+2 \xi) \operatorname{div} v
$$

which is reasonable in the case of weak deformations and when curl $v \wedge n$ $=0$ on the boundary. We will see that this modelization allows to obtain a priori estimates.

Afterwards, with the exception of $\mu$ and $\xi$, we take all the constants to be 1 (the case $\gamma=1$ is mathematically the most critical) and we study the problem $(\mathcal{P})=\{(\mathcal{F}),(\mathcal{S}),(\mathcal{C})\}$.

Remark 1.1. - If we do not take into account the variation of the domain occupied by the fluid, then $\Omega_{t}=\Omega_{0}$ and the conservation of the mass is not verified since

$$
\int_{\Omega_{t}} \rho(t)-\int_{\Omega_{0}} \rho_{0}=-\int_{\Sigma_{b}} \rho v \cdot n_{b} \mathrm{~d} \Sigma_{b} \neq 0 .
$$

By setting $\left.\Omega_{t}=\bigcup_{x \in \Omega_{p}}\right] u(x, t), 1[$ as the domain occupied by the fluid, we respect the assumption of conservation of mass. Indeed, by integrating the equation of continuity on $\Omega_{t}$, we find

$$
\int_{\Omega_{t}} \frac{\partial \rho}{\partial t}=-\int_{\Gamma_{b}} v \cdot n_{b} \rho \mathrm{d} \Gamma_{b}
$$


moreover, by using the Leibniz's formula, we obtain

$$
\int_{\Omega_{t}} \frac{\partial \rho}{\partial t}=\int_{\Omega_{p}} \int_{u(x, t)}^{1} \frac{\partial \rho}{\partial t}=\frac{\mathrm{d}}{\mathrm{d} t} \int_{\Omega_{t}} \rho+\int_{\Omega_{p}} \frac{\partial u}{\partial t} \rho \mathrm{d} \bar{x}
$$

and finally

$$
\frac{\mathrm{d}}{\mathrm{d} t} \int_{\Omega_{t}} \rho=-\int_{\Omega_{p}} \frac{\partial u}{\partial t} \rho \mathrm{d} \bar{x}-\int_{\Gamma_{b}} v \cdot n_{b} \rho \mathrm{d} \Gamma_{b},
$$

which, using (1.1), leads us to $\frac{\mathrm{d}}{\mathrm{d} t} \int_{\Omega_{t}} \rho=0$. This condition is physically correct and permits us to obtain a first estimate on $\rho$ in $L^{\infty}\left(0, T ; L^{1}\left(\Omega_{t}\right)\right)$ if $\rho_{0} \in L^{1}\left(\Omega_{0}\right)$.

Remark 1.2. - Afterwards, the definition of $\Omega_{t}$ and the modelization of the stress applied by the fluid to the plate allow to obtain the conservation of energy for the coupled system.

Remark 1.3. - The definition of the domain leads us to work with functional spaces defined over a family of domains dependent on time. In a previous work [4], we demonstrated that these spaces conserve the properties of the classical functional spaces.

Remark 1.4. $-\Gamma_{b}$ can be defined by the map $u: \Omega_{p} \mapsto \Gamma_{b}$. If $u(x, t)$ is continuous, then the boundary $\Gamma_{a} \cup \Gamma_{b}$ of $\Omega_{t}$ is continuous. Thus, as $v . n$ is defined on $\Gamma_{b}$ and $\partial u / \partial t$ on $\Omega_{p}$, we can assume that the function $v . n$ is defined on $\Omega_{p}$.

We show the existence of a weak solution for the coupled problem $(\mathcal{P})$ defined above. First, we present the estimates of energy associated with the system and we give a meaning to the trace of the stress terms. This allows us to pass to the limit in the plate equation. Finally, we construct approached solutions which satisfy the a priori estimates.

\section{AN EXISTENCE RESULT}

We give now the conditions which are sufficient to show the existence of a weak solution for the problem $(\mathcal{P})$. Let $\Theta \in] 0,1[, \varepsilon<1$ and the functions $f \in L^{1}\left(0, T ; H^{-1}\left(\Omega_{p}\right)\right), u_{0} \in H_{0}^{2}\left(\Omega_{p}\right), u_{1} \in H_{0}^{1}\left(\Omega_{p}\right)$, $v_{0} \in L^{2}\left(\Omega_{0}\right), \rho_{0} \in L^{1}\left(\Omega_{0}\right), \rho_{0} \log \rho_{0} \in L^{1}\left(\Omega_{0}\right)$ satisfying the following condition 


$$
\begin{aligned}
\Upsilon= & 2 \operatorname{meas}\left(\Omega_{t}\right)+(1-\varepsilon)\left\|u_{1}\right\|_{H_{0}^{1}\left(\Omega_{p}\right)}^{2}+\left\|\Delta u_{0}\right\|_{L^{2}\left(\Omega_{p}\right)}^{2}++\left\|v_{0}\right\|_{L^{2}\left(\Omega_{0}\right)}^{2} \\
& +2 \int_{\Omega_{0}} \rho_{0} \log \rho_{0}+\frac{2}{\varepsilon}\|f\|_{L^{1}\left(0, T ; H^{-1}\left(\Omega_{p}\right)\right)}^{2} \\
& <(1-\varepsilon) \Theta^{2}\left(\frac{\mu+\xi}{\mathbb{C}_{1}}\right)^{2}
\end{aligned}
$$

where $\mathbb{C}_{1}$ is a constant introduced below. If (2.1) is verified, then we have the following result

THEOREM 2.1. - There exists a solution $(u, v, \rho)$ of the above problem $(\mathcal{P})$ satisfying: $u \in W^{1, \infty}\left(0, T ; H_{0}^{1}\left(\Omega_{p}\right)\right) \cap L^{\infty}\left(0, T ; H_{0}^{2}\left(\Omega_{p}\right)\right), v \in$ $L^{\infty}\left(0, T ; L^{2}\left(\Omega_{t}\right)\right) \cap L^{2}\left(0, T ; H^{1}\left(\Omega_{t}\right)\right)$ and $\rho \in L^{\infty}\left(0, T ; L^{1}\left(\Omega_{t}\right)\right) \cap$ $L^{3 / 2}(Q)$ in such a way that the plate equation, the momentum and the continuity equations are respectively solved in $H^{-1}\left(0, T ; H^{-2}\left(\Omega_{p}\right)\right)$, $L^{3 / 2}\left(0, T ; W^{-1,3 / 2}\left(\Omega_{t}\right)\right)$ and $L^{12 / 11}\left(0, T ; W^{-1,1}\left(\Omega_{t}\right)\right)$.

\section{PROOF OF THEOREM 2.1}

\section{Step 1. A first a priori estimate}

Let $(u, v, \rho)$ be a solution of $(\mathcal{P})$, by multiplying the equation $(\mathcal{S})_{1}$ by $\partial u / \partial t$ and by multiplying the equation $(\mathcal{F})_{1}$ by $v$, we obtain successively

$$
\begin{aligned}
& \frac{1}{2} \frac{\mathrm{d}}{\mathrm{d} t}\left(\left\|\frac{\partial u}{\partial t}\right\|_{H_{0}^{1}\left(\Omega_{p}\right)}^{2}+\|\Delta u\|_{L^{2}\left(\Omega_{p}\right)}^{2}\right) \\
& =\int_{\Omega_{p}}(-\rho+(\mu+2 \xi) \operatorname{div} v) \frac{\partial u}{\partial t}+\int_{\Omega_{p}} f \frac{\partial u}{\partial t} \\
& \frac{1}{2} \int_{\Omega_{t}} \frac{\partial v^{2}}{\partial t}+(\mu+\xi)\|v\|_{H^{1}\left(\Omega_{t}\right)}^{2}+\xi\|\operatorname{div} v\|_{L^{2}\left(\Omega_{t}\right)}^{2} \\
& =\int_{\Omega_{t}} \rho \operatorname{div} v+\int_{\Gamma_{b}}(-\rho+(\mu+2 \xi) \operatorname{div} v) v \cdot n_{b} .
\end{aligned}
$$

Let us first recall that in Eq. (2.3), the domain $\Omega_{t}$ depends on time, therefore:

$$
\frac{1}{2} \int_{\Omega_{t}} \frac{\partial v^{2}}{\partial t}=\frac{1}{2} \int_{u(t)}^{1} \int_{\Omega_{p}} \frac{\partial v^{2}}{\partial t}=\frac{1}{2} \frac{\mathrm{d}}{\mathrm{d} t}\|v\|_{L^{2}\left(\Omega_{t}\right)}^{2}+\frac{1}{2} \int_{\Omega_{p}} v^{2} \frac{\partial u}{\partial t} .
$$


Moreover, still in Eq. (2.3), we remark that formally

$$
\begin{aligned}
\int_{\Omega_{t}} \rho \operatorname{div} v & =-\int_{\Omega_{t}} \frac{\nabla \rho}{\rho}(v \rho)+\int_{\Gamma_{b}} \rho v \cdot n_{b} \\
& =\int_{\Omega_{t}} \log \rho \operatorname{div}(v \rho)+\int_{\Gamma_{b}}(\rho-\rho \log \rho) v \cdot n_{b},
\end{aligned}
$$

consequently by using the continuity equation

$$
\begin{aligned}
\int_{\Omega_{t}} \rho \operatorname{div} v & =-\int_{\Omega_{t}} \frac{\partial \rho}{\partial t} \log \rho+\int_{\Gamma_{b}}(\rho-\rho \log \rho) v \cdot n_{b} \\
& =\int_{\Omega_{t}} \frac{\partial}{\partial t}(\rho-\rho \log \rho)+\int_{\Gamma_{b}}(\rho-\rho \log \rho) v \cdot n_{b},
\end{aligned}
$$

and so with the Leibniz's formula, we obtain

$$
\begin{aligned}
\int_{\Omega_{t}} \rho \operatorname{div} v= & -\frac{\mathrm{d}}{\mathrm{d} t} \int_{\Omega_{t}} \rho \log \rho+\int_{\Omega_{p}}(\rho-\rho \log \rho) \frac{\partial u}{\partial t} \\
& +\int_{\Gamma_{b}}(\rho-\rho \log \rho) v \cdot n_{b} .
\end{aligned}
$$

Thus, by using Eq. (2.4) and Eq. (2.6) in Eq. (2.3), we find

$$
\begin{aligned}
\frac{1}{2} \frac{\mathrm{d}}{\mathrm{d} t}\left(\|v\|_{L^{2}\left(\Omega_{t}\right)}^{2}+2 \int_{\Omega_{t}} \rho \log \rho\right)+(\mu+\xi)\|v\|_{H^{1}\left(\Omega_{t}\right)}^{2}+\xi\|\operatorname{div} v\|_{L^{2}\left(\Omega_{t}\right)}^{2} \\
=-\frac{1}{2} \int_{\Omega_{p}} v^{2} \frac{\partial u}{\partial t}-\int_{\Omega_{p}}(\rho-\rho \log \rho) \frac{\partial u}{\partial t}+\int_{\Gamma_{b}}(\rho-\rho \log \rho) v \cdot n_{b} \\
\quad+\int_{\Gamma_{b}}(-\rho+(\mu+2 \xi) \operatorname{div} v) v \cdot n_{b} .
\end{aligned}
$$

Finally, considering that

$$
-\frac{1}{2} \int_{\Omega_{p}} v^{2} \frac{\partial u}{\partial t} \leqslant \mathbb{C}_{1}\left\|\frac{\partial u}{\partial t}\right\|_{L^{\infty}\left(0, T ; L^{2}\left(\Omega_{p}\right)\right)}\|v\|_{L^{2}\left(0, T ; H^{1}\left(\Omega_{t}\right)\right)}^{2}
$$

and (1.1)

$$
\int_{\Gamma_{b}} \sigma v \cdot n_{b} \mathrm{~d} \Gamma_{b}=-\int_{\Omega_{p}} \sigma \frac{\partial u}{\partial t} \mathrm{~d} \bar{x}
$$


by summing Eqs. (2.2) and (2.7), we obtain the following energy estimate

$$
\begin{aligned}
& 2 \operatorname{meas}\left(\Omega_{t}\right)+(1-\varepsilon)\left\|\frac{\partial u(t)}{\partial t}\right\|_{H_{0}^{1}\left(\Omega_{p}\right)}^{2}+\|\Delta u(t)\|_{L^{2}\left(\Omega_{p}\right)}^{2}+\|v(t)\|_{L^{2}\left(\Omega_{t}\right)}^{2} \\
& \quad+2 \int_{\Omega_{t}} \rho(t) \log \rho(t)+2\left((\mu+\xi)-\mathbb{C}_{1}\left\|\frac{\partial u}{\partial t}\right\|_{L^{\infty}\left(0, T ; L^{2}\left(\Omega_{p}\right)\right)}\right) \\
& \quad \times\|v\|_{L^{2}\left(0, T ; H^{1}\left(\Omega_{t}\right)\right)}^{2}+2 \xi\|\operatorname{div} v\|_{L^{2}(Q)}^{2} \leqslant \Upsilon
\end{aligned}
$$

where $\Upsilon$ is defined by Eq. (2.1) and $\int_{\Omega_{t}} \rho(t) \log \rho(t)+\operatorname{meas}\left(\Omega_{t}\right) \geqslant 0$.

Assuming that

$$
(\mu+\xi)-\mathbb{C}_{1}\left\|\frac{\partial u}{\partial t}\right\|_{L^{\infty}\left(0, T ; L^{2}\left(\Omega_{p}\right)\right)} \geqslant 0,
$$

we obtain $u$ bounded in $W^{1, \infty}\left(0, T ; H_{0}^{1}\left(\Omega_{p}\right)\right) \cap L^{\infty}\left(0, T ; H_{0}^{2}\left(\Omega_{p}\right)\right) \subset$ $C^{0}\left(\bar{Q}_{p}\right), v$ bounded in $L^{\infty}\left(0, T ; L^{2}\left(\Omega_{t}\right)\right) \cap L^{2}\left(0, T ; H^{1}\left(\Omega_{t}\right)\right), \rho$ and $\rho \log \rho$ bounded in $L^{\infty}\left(0, T ; L^{1}\left(\Omega_{t}\right)\right)$. We show, if the condition of "small data" (2.1) is verified, that (2.10) is always true.

Looking at (2.9), it is clear that we can obtain estimates on $u$ and $\rho$ if and only if the condition (2.10) is verified for each $t$, that is to say if

$$
\left\|\frac{\partial u(t)}{\partial t}\right\|_{L^{2}\left(\Omega_{p}\right)} \leqslant \frac{\mu+\xi}{\mathbb{C}_{1}}
$$

which signifies that $\partial u / \partial t$ must stay in the ball of $L^{2}\left(\Omega_{p}\right)$ with center 0 and radius $(\mu+\xi) / \mathbb{C}_{1}$. To prove this point, we use the assumption (2.1) based on the data. Since $\partial u / \partial t$ is continue from $[0, \mathrm{~T}]$ in $L^{2}\left(\Omega_{p}\right)$ and

$$
\left\|\frac{\partial u(0)}{\partial t}\right\|_{L^{2}\left(\Omega_{p}\right)}=\left\|u_{1}\right\|_{L^{2}\left(\Omega_{p}\right)} \leqslant \Theta \frac{\mu+\xi}{\mathbb{C}_{1}},
$$

then there exists $t^{\prime}<T$ such that

$$
\left\|\frac{\partial u\left(t^{\prime}\right)}{\partial t}\right\|_{L^{2}\left(\Omega_{p}\right)} \leqslant \Theta \frac{\mu+\xi}{\mathbb{C}_{1}} .
$$

By setting $t_{1}$ as the smaller time such that,

$$
\left\|\frac{\partial u\left(t_{1}\right)}{\partial t}\right\|_{L^{2}\left(\Omega_{p}\right)}=\Theta \frac{\mu+\xi}{\mathbb{C}_{1}},
$$


estimate (2.9) written at time $t_{1}$ leads us to

$$
(1-\varepsilon) \Theta^{2}\left(\frac{\mu+\xi}{\mathbb{C}_{1}}\right)^{2} \leqslant \Upsilon<(1-\varepsilon) \Theta^{2}\left(\frac{\mu+\xi}{\mathbb{C}_{1}}\right)^{2}
$$

which contradicts the assumption (2.1). Therefore, $\partial u(t) / \partial t$ stays in the stability ball whatever $t \in[0, T]$.

Remark 2.1. - If $u$ is bounded in $W^{1, \infty}\left(0, T ; H_{0}^{1}\left(\Omega_{p}\right)\right) \cap L^{\infty}(0, T$; $\left.H_{0}^{2}\left(\Omega_{p}\right)\right)$ then $\frac{\partial u}{\partial t} \cos \left(\omega_{3}\right)$ is bounded in $L^{\infty}\left(0, T ; W^{1,2 q /(4+q)}\left(\Omega_{p}\right)\right)$ with $4 \leqslant q<\infty$. Indeed,

$$
\frac{\partial \cos \left(\omega_{3}\right)}{\partial x_{i}}=2\left(\sum_{j=1}^{2} \frac{\partial u}{\partial x_{j}} \frac{\partial^{2} u}{\partial x_{i} \partial x_{j}}\right) \times \frac{1}{\left(1+\left(\frac{\mathrm{d} u}{\mathrm{~d} \bar{x}}\right)^{2}\right)}
$$

then

$$
\begin{aligned}
\frac{\partial \cos \left(\omega_{3}\right)}{\partial x_{i}} & \in L^{\infty}\left(0, T ; H_{0}^{1}\left(\Omega_{p}\right)\right) \times L^{\infty}\left(0, T ; L^{2}\left(\Omega_{p}\right)\right) \times L^{\infty}(Q) \\
& \subset L^{\infty}\left(0, T ; L^{2 q /(2+q)}\left(\Omega_{p}\right)\right),
\end{aligned}
$$

thus

$$
\begin{aligned}
& \frac{\partial u}{\partial t} \cos \left(\omega_{3}\right) \\
& \quad \in L^{\infty}\left(0, T ; H_{0}^{1}\left(\Omega_{p}\right)\right) \times L^{\infty}(Q) \cap L^{\infty}\left(0, T ; W^{1,2 q /(2+q)}\left(\Omega_{p}\right)\right) \\
& \quad \subset L^{\infty}\left(0, T ; W^{1,2 q /(4+q)}\left(\Omega_{p}\right)\right)
\end{aligned}
$$

\section{Step 2. Weak formulation}

The problem is solved in a weak meaning. In particular, let us specify the weak formulation associated to the fluid. We homogenize the momentum equation by setting $v=w+\bar{w}$ where $\bar{w}=\nabla \bar{h}$ and $\bar{h}$ is the solution of

$$
\begin{cases}\frac{\partial \bar{h}}{\partial t}-(\mu+2 \xi) \Delta \bar{h}=0, & \text { in } Q \\ \nabla \bar{h} \cdot n_{a}=0, & \text { on } \Sigma_{a} \\ \nabla \bar{h} \cdot n_{b}=\frac{\partial u}{\partial t} \cos \left(\omega_{3}\right) \in L^{\infty}\left(0, T ; W^{1,2 q /(4+q)}\left(\Omega_{p}\right)\right), & \text { on } \Sigma_{b} . \\ h(t=0)=h_{0}(x) \in H^{1}\left(\Omega_{0}\right), \rho(t=0)=\rho_{0}(x) \geqslant 0, & \text { in } \Omega_{0}\end{cases}
$$


In fact, afterwards, $\frac{\partial u}{\partial t} \cos \left(\omega_{3}\right)$ is fixed and we solve the problem by using a fixed point method. Thus, we obtain the following homogeneous problem

$$
\left(\mathcal{F}^{\prime}\right) \begin{cases}\frac{\partial w}{\partial t}-(\mu+\xi) \Delta w-\xi \nabla \operatorname{div} w+\nabla \rho=0, & \text { in } Q, \\ \frac{\partial \rho}{\partial t}+\operatorname{div}(w \rho)=-\operatorname{div}(\bar{w} \rho), \rho \geqslant 0, & \text { in } Q, \\ \operatorname{curl} w \wedge n_{i}=0, i \in\{a ; b\}, & \text { on } \Sigma, \\ w . n_{i}=0, i \in\{a ; b\}, & \text { on } \Sigma, \\ w(t=0)=v_{0}(x)-\nabla \bar{h}_{0}(x), \rho(t=0)=\rho_{0}(x) \geqslant 0, & \text { in } \Omega_{0},\end{cases}
$$

which is solved in a weak meaning. If we set $W=\left\{\phi \in H^{1}\left(\Omega_{t}\right), \phi . n=0\right.$ on $\Gamma\}$ and $\mathrm{a}(w, \phi)=(\operatorname{div} w, \operatorname{div} \phi)+(\operatorname{curl} w, \operatorname{curl} \phi)$ where $(.,$.$) is the$ scalar product in $L^{2}\left(\Omega_{t}\right)$, the problem is:

Find $w \in L^{2}(0, T ; W) \cap L^{\infty}\left(0, T ; L^{2}\left(\Omega_{t}\right)\right)$ and $\rho \in L^{3 / 2}(Q) \cap L^{\infty}(0$, $\left.T ; L^{1}\left(\Omega_{t}\right)\right)$ satisfying

$$
\left\{\begin{array}{c}
\int_{0}^{T}\left\langle\frac{\partial w}{\partial t}, \phi\right\rangle_{W^{\prime}, W}+(\mu+\xi) \int_{0}^{T} \mathrm{a}(w, \phi)+\xi \int_{0}^{T}(\operatorname{div} w, \operatorname{div} \phi) \\
\quad+\int_{0}^{T}(\rho, \operatorname{div} \phi)=0, \quad \forall \phi \in L^{3}\left(0, T ; W \cap W^{1,3}\left(\Omega_{t}\right)\right), \\
\frac{\partial \rho}{\partial t}+\operatorname{div}(w \rho)=-\operatorname{div}(\bar{w} \rho), \quad \text { in } L^{12 / 11}\left(0, T ; W^{-1,1}\left(\Omega_{t}\right)\right), \\
w(t=0)=v_{0}(x)-\nabla \bar{h}_{0}(x), \rho(t=0)=\rho_{0}(x) \geqslant 0, \quad \text { in } \Omega_{0} .
\end{array}\right.
$$

Note that the plate equation is solved in the following meaning

$$
\frac{\partial^{2} u}{\partial t^{2}}-\Delta \frac{\partial^{2} u}{\partial t^{2}}+\Delta^{2} u=f-\rho+(\mu+2 \xi) \operatorname{div} v \in H^{-1}\left(0, T ; H^{-2}\left(\Omega_{p}\right)\right) .
$$

\section{Step 3. An additional estimate on $\rho$}

The estimates obtained on $\rho$ in the "step 1" are not sufficient to pass to the limit in the continuity equation in the three-dimensional situation. We show below how to obtain the estimate $\rho$ bounded in $L^{3 / 2}(Q)$ which is sufficient to pass to the limit. 
Let $w_{1}$ and $w_{2}$ be the unique functions satisfying $w=w_{1}+w_{2}$ with $\operatorname{div} w_{2}=0$ and $\operatorname{rot} w_{1}=0$. We note that this notation leads us to

$$
\begin{gathered}
\frac{\partial w_{1}}{\partial t}-(\mu+2 \xi) \nabla\left(\operatorname{div} w_{1}\right)+\nabla \rho=-\frac{\partial \bar{w}}{\partial t}-(\mu+2 \xi) \nabla(\operatorname{div} \bar{w})=0, \\
\frac{\partial w_{2}}{\partial t}-\mu \operatorname{Rot}\left(\operatorname{rot} w_{2}\right)=0,
\end{gathered}
$$

with $\operatorname{rot} w_{2}=0$ and $w_{1} \cdot n=0$ on $\Sigma$. Moreover, we have $w_{1}=\nabla h$ where $h$ satisfies

$$
\begin{aligned}
\frac{\partial h}{\partial t}-(\mu+2 \xi) \Delta h+\rho & =\int_{\Omega_{0}}-\rho_{0}+\frac{1}{\operatorname{meas}\left(\Omega_{t}\right)} \int_{\Omega_{p}} h \frac{\partial u}{\partial t} \\
& =\alpha(t) \in L^{\infty}(0, T) .
\end{aligned}
$$

We also have (formally at least)

$$
\frac{\partial \sqrt{\rho}}{\partial t}+\operatorname{div}(\sqrt{\rho} v)-\frac{1}{2} \operatorname{div}(v) \sqrt{\rho}=0 .
$$

We multiply (2.13) by $h$ and we integrate on $Q$, we obtain

$$
\begin{gathered}
\int_{Q} \frac{\partial \sqrt{\rho}}{\partial t} h-\frac{1}{2} \int_{Q}(\nabla h)^{2} \sqrt{\rho}-\frac{1}{4} \int_{Q} \Delta h^{2} \sqrt{\rho}-\int_{Q} \bar{w} \nabla h \sqrt{\rho} \\
-\frac{1}{2} \int_{Q} \operatorname{div}(\bar{w}) h \sqrt{\rho}+\int_{\Sigma} v \cdot n h \sqrt{\rho}=0,
\end{gathered}
$$

moreover (2.12) gives us

$$
\int_{Q} \frac{\partial h}{\partial t} \sqrt{\rho}-(\mu+\xi) \int_{Q} \Delta h \sqrt{\rho}+\int_{Q} \rho^{3 / 2}=\int_{0}^{T} \alpha(t) \int_{\Omega_{t}} \sqrt{\rho} .
$$

Thus, by summing (2.14)-(2.15) and by using Leibniz's formula, the terms on the boundary are cancelled thanks to the condition (1.1) and we obtain the following equation

$$
\begin{aligned}
\|\rho\|_{L^{3 / 2}(Q)}^{3 / 2}= & \frac{1}{2} \int_{Q}(\nabla h)^{2} \sqrt{\rho}+\frac{1}{4} \int_{Q} \Delta h^{2} \sqrt{\rho}+\int_{Q} \bar{w} \nabla h \sqrt{\rho} \\
& +\frac{1}{2} \int_{Q} \operatorname{div}(\bar{w}) h \sqrt{\rho}-\int_{\Omega_{t}} h \sqrt{\rho}+\int_{\Omega_{0}} h_{0} \sqrt{\rho_{\delta 0}}
\end{aligned}
$$




$$
+(\mu+\xi) \int_{Q} \Delta h \sqrt{\rho}+\int_{0}^{T} \alpha(t) \int_{\Omega_{t}} \sqrt{\rho} .
$$

In order to estimate the right hand side term of (2.16) let us recall that $h$ is bounded in $L^{\infty}\left(0, T ; H^{1}\left(\Omega_{t}\right)\right) \cap L^{2}\left(0, T ; H^{2}\left(\Omega_{t}\right)\right)$ which by using the harmonic mean, leads us to $h$ bounded in $L^{4}\left(\left(0, T ; W^{1,3}\left(\Omega_{t}\right)\right)\right.$. In addition, $H^{2}\left(\Omega_{t}\right)$ is an algebra therefore $h^{2}$ is bounded in $L^{1}\left(0, T ; H^{2}\left(\Omega_{t}\right)\right)$. Lastly, let us recall that $\sqrt{\rho}$ is bounded in $L^{\infty}\left(0, T ; L^{2}\left(\Omega_{t}\right)\right)$ since according to Remark 1.1, $\rho$ is bounded in $L^{\infty}\left(0, T ; L^{1}\left(\Omega_{t}\right)\right)$. Therefore, the different terms are estimated as follows

$$
\begin{aligned}
& \frac{1}{2} \int_{Q}(\nabla h)^{2} \sqrt{\rho} \leqslant \mathbb{C}_{\varepsilon}\|\nabla h\|_{L_{t}^{4}\left(L_{x}^{3}\right)}^{3}+\varepsilon\|\rho\|_{L^{3 / 2}(Q)}^{3 / 2} \leqslant \mathbb{C}+\varepsilon\|\rho\|_{L^{3 / 2}(Q)}^{3 / 2}, \\
& \int_{\Omega_{t}} h \sqrt{\rho} \leqslant \mathbb{C}\|h\|_{L^{2}(Q)}\|\sqrt{\rho}\|_{L^{2}(Q)} \leqslant \mathbb{C}, \\
& \frac{1}{4} \int_{\Omega_{t}} \Delta h^{2} \sqrt{\rho} \leqslant \mathbb{C}\left\|\Delta h^{2}\right\|_{L_{t}^{1}\left(L_{x}^{2}\right)}\|\sqrt{\rho}\|_{L_{t}^{\infty}\left(L_{x}^{2}\right)} \leqslant \mathbb{C}, \\
& \int_{Q} \bar{w} \nabla h \sqrt{\rho} \leqslant \mathbb{C}\|\bar{w}\|_{L_{t}^{12 / 5}\left(L_{x}^{3}\right)}\|\nabla h\|_{L_{t}^{4}\left(L_{x}^{3}\right)}\|\rho\|_{L^{3} 2(Q)}^{1 / 2} \\
& \leqslant \mathbb{C}_{\varepsilon}\|\bar{w}\|_{L_{t}^{12 / 5}\left(L_{x}^{3}\right)}^{3 / 2}\|\nabla h\|_{L_{t}^{4}\left(L_{x}^{3}\right)}^{3 / 2}+\varepsilon\|\rho\|_{L^{3 / 2}(Q)}^{3 / 2} \\
& \leqslant \mathbb{C}\|\bar{w}\|_{L_{t}^{12 / 5}\left(L_{x}^{3}\right)}^{3 / 2}+\varepsilon\|\rho\|_{L^{3 / 2}(Q)}^{3 / 2}, \\
& \int_{Q} \operatorname{div} \bar{w} h \sqrt{\rho} \leqslant \mathbb{C}\|\operatorname{div} \bar{w}\|_{L_{t}^{3 / 2}\left(L_{x}^{2}\right)}\|h\|_{L_{t}^{\infty}\left(L_{x}^{6}\right)}\|\rho\|_{L^{3 / 2}(Q)}^{1 / 2} \\
& \leqslant \mathbb{C}_{\varepsilon}\|\operatorname{div} \bar{w}\|_{L_{t}^{3 / 2}\left(L_{x}^{2}\right)}^{3 / 2}\|h\|_{L_{t}^{\infty}\left(L_{x}^{6}\right)}^{3 / 2}+\varepsilon\|\rho\|_{L^{3 / 2}(Q)}^{3 / 2} \\
& \leqslant \mathbb{C}\|\operatorname{div} \bar{w}\|_{L_{t}^{3 / 2}\left(L_{x}^{2}\right)}^{3 / 2}+\varepsilon\|\rho\|_{L^{3 / 2}(Q)}^{3 / 2}, \\
& (\mu+\xi) \int_{Q} \Delta h \sqrt{\rho} \leqslant \mathbb{C}\|\Delta h\|_{L^{2}(Q)}\|\sqrt{\rho}\|_{L^{2}(Q)} \leqslant \mathbb{C}
\end{aligned}
$$




$$
\int_{0}^{T} \alpha(t) \int_{Q} \sqrt{\rho} \leqslant \mathbb{C}\|\alpha(t)\|_{L^{1}(0, T)}\|\sqrt{\rho}\|_{L_{t}^{\infty}\left(L_{x}^{1}\right)} \leqslant \mathbb{C} .
$$

Finally, by using (2.17)-(2.23) in (2.16), we obtain

$$
(1-3 \varepsilon)\|\rho\|_{L^{3 / 2}(Q)}^{3 / 2} \leqslant \mathbb{C}\left(1+\|\bar{w}\|_{L_{t}^{12 / 5}\left(L_{x}^{3}\right)}^{3 / 2}+\|\operatorname{div} \bar{w}\|_{L_{t}^{3 / 2}\left(L_{x}^{2}\right)}^{3 / 2}\right),
$$

where $\bar{w}=\nabla \bar{h}$ and with $\bar{h}$ the solution of problem $(\mathcal{R})$ in which $\frac{\partial u}{\partial t} \cos \left(\omega_{3}\right) \in L^{\infty}\left(0, T ; W^{1,2 q /(4+q)}\left(\Omega_{p}\right)\right)$ with $0 \leqslant q<\infty$. Consequently, $\bar{w} \in L^{\infty}\left(0, T ; L^{2}\left(\Omega_{t}\right)\right) \cap L^{2}\left(0, T ; H^{1}\left(\Omega_{t}\right)\right) \subset L^{4}\left(0, T ; L^{3}\left(\Omega_{t}\right)\right)$ and thus

$$
\|\rho\|_{L^{3 / 2}(Q)} \leqslant \mathcal{K}
$$

\section{Step 4. Passage to the limit}

Let $\left(u_{\mu}, v_{\mu}, \rho_{\mu}\right)$ be a sequence of approached solutions satisfying the estimates (2.9) and (2.24). We can extract a subsequence which is still noted $\left(u_{\mu}, v_{\mu}, \rho_{\mu}\right)$ such that

$$
\begin{gathered}
u_{\mu} \stackrel{\text { weak* }}{\longrightarrow} \hat{u} \quad \text { in } L^{\infty}\left(0, T ; H_{0}^{1}\left(\Omega_{p}\right)\right), \\
u_{\mu} \stackrel{\text { weak* }}{\longrightarrow} \hat{u} \quad \text { in } W^{1, \infty}\left(0, T ; H_{0}^{2}\left(\Omega_{p}\right)\right), \\
\rho_{\mu} \stackrel{\text { weak }}{\longrightarrow} \hat{\rho} \quad \text { in } L^{3 / 2}(Q), \\
v_{\mu} \stackrel{\text { weak* }}{\longrightarrow} \hat{v} \quad \text { in } L^{\infty}\left(0, T ; L^{2}\left(\Omega_{t}\right)\right), \\
v_{\mu} \stackrel{\text { weak }}{\longrightarrow} \hat{v} \quad \text { in } L^{2}\left(0, T ; H^{1}\left(\Omega_{t}\right)\right) .
\end{gathered}
$$

The passage to the limit presents three points on which we want to insist: the passage to the limit in the continuity equation and the ones concerning respectively the terms of the stress in the plate equation and the coupling condition.

In order to pass to the limit in the continuity equation, we need to check that Aubin's theorem is always true for a the spaces $L^{p}(0, T ; X(t))$. The others difficulties can be solved by an adaptation of the demonstration given in [6] or [7].

We consider $\Theta_{n}$ a bounded sequence in $L^{p_{0}}\left(0, T ; H^{m_{0}}\left(\Omega_{t}\right)\right)$ such that $\partial \Theta_{n} / \partial t$ is bounded in $L^{p_{1}}\left(0, T ; H^{-m_{1}}\left(\Omega_{t}\right)\right)$ with $1<p_{0}, p_{1}<\infty$ and 
$m_{0}>m_{1}>0$. We extend the sequence $\Theta_{n}$ by using the extension operator of $H^{m_{0}+1}(Q)$ in $H^{m_{0}+1}\left(\mathbb{R}^{4}\right)$. We note that the support of the functions is contained in a compact of $\mathbb{R}^{4}$ if $Q$ is compact. Let $\bar{\Theta}_{n}$ be the extension of $\Theta_{n}$, we note $\Omega^{\prime}=\bigcup_{t \in] 0, T[} \Omega_{t}$, then $\bar{\Theta}_{n}$ is bounded in $L^{p_{0}}\left(0, T ; H^{m_{0}}\left(\Omega^{\prime}\right)\right.$ and $\partial \bar{\Theta}_{n} / \partial t$ is bounded in $L^{p_{1}}\left(0, T ; H^{-m_{1}}\left(\Omega^{\prime}\right)\right.$ ) (see for example [5]). This result is sufficient to apply Aubin's compactness lemma.

Concerning the equation of the structure, the main difficulty is the passage to the limit in the trace terms. We note that

$$
\nabla\left\{(\mu+2 \xi) \operatorname{div}\left(w_{1}+\bar{w}\right)-\rho\right\}=\frac{\partial w_{1}}{\partial t}+\frac{\partial \bar{w}}{\partial t} \in H^{-1}\left(0, T ; H^{1}\left(\Omega_{t}\right)\right),
$$

hence, $\gamma((\mu+2 \xi) \operatorname{div} v-\rho) \in H^{-1}\left(0, T ; H^{3 / 2}(\Gamma)\right)$ and

$$
\gamma\left\{\rho_{\mu}-(\mu+2 \xi) \operatorname{div} v_{\mu}\right\} \rightarrow \Theta \quad \text { in } H^{-1}\left(0, T ; H^{3 / 2}(\Gamma)\right) \text { weakly, }
$$

which allows us to give a meaning to the trace of the sum $(\rho-(\mu+$ $2 \xi$ ) div $v$ ). In order to give a meaning to each term of this sum, we define $\Xi_{\mu}$ and $\Xi$ as the vectors

$$
\Xi_{\mu}=\left\{\begin{array}{c}
\rho_{\mu} \\
\rho_{\mu} v_{1 \mu} \\
\rho_{\mu} v_{2 \mu} \\
\rho_{\mu} v_{3 \mu}
\end{array}\right\} \quad \text { and } \quad \Xi=\left\{\begin{array}{c}
\rho \\
\rho v_{1} \\
\rho v_{2} \\
\rho v_{3}
\end{array}\right\} \text {. }
$$

We obtain $\Xi_{\mu} \in L^{1}(Q)^{4}$ and $\operatorname{div}_{x, t} \Xi_{\mu}=0=\partial \rho_{\mu} / \partial t+\operatorname{div}\left(\rho_{\mu} v_{\mu}\right) \in$ $L^{1}(Q)$, therefore $\Xi_{\mu} \in L_{\text {div }}^{1}(Q)=\left\{\phi \in L^{1}(Q)^{4}\right.$, div $\left.\phi \in L^{1}(Q)\right\}$ and thanks to the passage to the limit in the equation of continuity, we have $\Xi_{\mu} \rightarrow \Xi$ in $L_{\text {div }}^{1}(Q)$-weak. Thus, according to the continuity of the trace application [1], we have $\gamma\left(\Xi_{\mu}\right) \rightarrow \gamma(\Xi)$ in $\left(W^{1, \infty}(\partial Q)\right)^{\prime}$ weakly and in particular

$$
\gamma\left(\rho_{\mu}\right) \rightarrow \gamma(\rho) \text { in }\left(W^{1, \infty}(\partial Q)\right)^{\prime} \text { weakly. }
$$

Finally, from (2.25) and (2.26), we obtain

$$
\gamma\left(\operatorname{div} v_{\mu}\right) \rightarrow \gamma(\operatorname{div} v) \quad \text { in } \mathcal{D}^{\prime}(\partial Q)
$$

To pass to the limit in the coupling condition, we use the fact that $u$ is bounded in $W^{1, \infty}\left(0, T ; H_{0}^{1}\left(\Omega_{p}\right)\right) \cap L^{\infty}\left(0, T ; H_{0}^{2}\left(\Omega_{p}\right)\right)$. Indeed, we 
deduce of this estimate that

$$
\frac{\partial \cos \left(\omega_{3}\right)}{\partial t}=2\left(\sum_{j=1}^{2} \frac{\partial u}{\partial x_{j}} \frac{\partial^{2} u}{\partial t \partial x_{j}}\right) \times \frac{1}{\left(1+(\mathrm{d} u / \mathrm{d} \bar{x})^{2}\right)}
$$

is bounded in $L^{\infty}\left(0, T ; L^{2-\varepsilon}\left(\Omega_{p}\right)\right.$ and the bound of $\frac{\partial u}{\partial t} \cos \left(\omega_{3}\right)$ in $L^{1}(Q)$ permits us to show that $\frac{\partial u}{\partial t} \cos \left(\omega_{3}\right)$ converges in the sense of distributions on $\Omega_{p} \times(0, T)([6]$ Chapter 5, Lemma 5.1).

\section{Step 5. Construction of approached solutions}

In order to construct some approached solutions which satisfy the $a$ priori estimates, we use a fixed point technique. More precisely, we first solve the fluid equations by setting $v . n=\partial g / \partial t \in H^{1}\left(0, T ; H^{1}\left(\Omega_{p}\right)\right)$, then we solve the plate equation. We show that we can define a map $\Pi$ such that

$$
\begin{gathered}
\Pi: v . n_{b}=\frac{\partial g}{\partial t} \in H^{1}\left(0, T ; H^{1}\left(\Omega_{p}\right)\right) \\
\mapsto \frac{\partial u}{\partial t} \cos \left(\omega_{3}\right) \in H^{1}\left(0, T ; H^{1}\left(\Omega_{p}\right)\right),
\end{gathered}
$$

and $\Pi$ satisfies the criteria of the Kakutani's fixed point theorem which permits us to demonstrate that $\Pi$ admits a fixed point $\partial g / \partial t \in$ $H^{1}\left(0, T ; H^{1}\left(\Omega_{p}\right)\right)$ such that the coupling condition is satisfied

$$
v \cdot n_{b}=\frac{\partial g}{\partial t}=\frac{\partial u}{\partial t} \cos \left(\omega_{3}\right) \in H^{1}\left(0, T ; H^{1}\left(\Omega_{p}\right)\right) .
$$

We detail this point below (see also [3] and [4]).

\subsection{Resolution of the fluid equations}

(a) Homogenization of the fluid equation. First of all, we homogenize the momentum equation. With this aim in view, we set $v=w+\bar{w}$ where $\bar{w}=\nabla \bar{h}$ with $\bar{h}$ the solution to the following problem

$$
(\mathcal{R}) \quad\left\{\begin{array}{ll}
-\Delta \bar{h}(t)=F(t) \in H^{1}\left(0, T ; L^{\infty}\left(\Omega_{t}\right)\right), & \text { in } Q, \\
\nabla \bar{h} \cdot n_{a}=0, & \text { on } \Sigma_{a}, \\
\nabla \bar{h} \cdot n_{b}=\frac{\partial g}{\partial t} \in H^{1}\left(0, T ; H^{1}\left(\Omega_{p}\right)\right), & \text { on } \Sigma_{b} .
\end{array}\right\}
$$


where $F(t)$ is such that $\int_{\Omega_{t}} F(t)+\int_{\Gamma_{b}} \partial g / \partial t=0$. Thus $\bar{w} \in H^{1}(0, T$; $\left.H^{3 / 2}\left(\Omega_{t}\right)\right)$ and $\operatorname{div} \bar{w}=\Delta \bar{h} \in H^{1}\left(0, T ; L^{\infty}\left(\Omega_{t}\right)\right)$, moreover for all $t$ we have the estimate

$$
\|\operatorname{div} \bar{w}(t)\|_{H^{1 / 2}\left(\Omega_{t}\right)}^{2} \leqslant \mathbb{C}\left(\|F(t)\|_{L^{\infty}\left(\Omega_{t}\right)}^{2}+\left\|\frac{\partial g}{\partial t}(t)\right\|_{H^{1}\left(\Omega_{p}\right)}^{2}\right) .
$$

We solve the problem $(\mathcal{R})$ with the Galerkin method by using a basis sufficiently smooth (for example a basis of $H^{5}(Q)$ ). The act of working on $Q$ allows us to circumvent the difficulties linked to the variations of the domain $\Omega_{t}$. We then solve the fluid equations by setting $w=v-\bar{w}$. Thus, we obtain the homogeneous problem $\left(\mathcal{F}^{\prime}\right)$.

(b) Regularisation of the homogeneous problem. We solve the homogeneous momentum equation by using an elliptical smoothing technique. Furthermore, we make a $L^{2}$-regularization of the continuity equation which is necessary to construct solutions satisfying (2.13). We therefore must solve the following problem

$$
\left(\mathcal{F}_{\delta \varepsilon}^{\prime}\right) \begin{cases}-\varepsilon \frac{\partial^{2} w_{\varepsilon}}{\partial t^{2}}+\frac{\partial w_{\varepsilon}}{\partial t}-(\mu+\xi) \Delta w_{\varepsilon} & \\ -\xi \nabla \operatorname{div} w_{\varepsilon}+\nabla \rho_{\delta}=\nabla \bar{F}, & \text { in } Q, \\ \nabla h_{\varepsilon} \cdot n=0, & \text { on } \Sigma, \\ \operatorname{curl} w_{\varepsilon}=0, & \text { on } \Sigma, \\ \frac{\partial w_{\varepsilon}}{\partial t}=0, & \text { on } \Sigma, \\ w_{\varepsilon}(t=0) \rightarrow w_{0} \text { as }\{\delta, \varepsilon\} \rightarrow 0, & \text { in } L^{2}\left(\Omega_{0}\right), \\ \frac{\partial w_{\varepsilon}(0)}{\partial t}=0, & \text { in } \Omega_{0}, \\ \frac{\partial w_{\varepsilon}(T)}{\partial t}=0, & \text { in } \Omega_{T}, \\ \frac{\partial \rho_{\delta}}{\partial t}+\operatorname{div}\left(\rho_{\delta} w_{\varepsilon}\right)+\operatorname{div}\left(\rho_{\delta} \bar{w}\right)+\delta \rho_{\delta}^{2}=0, \rho_{\delta} \geqslant 0, & \text { in } Q, \\ \rho_{\delta}(0) \rightarrow \rho_{0} \text { as }\{\delta, \varepsilon\} \rightarrow 0, & \text { in } L^{1}\left(\Omega_{0}\right), \\ \rho_{\delta}(0) \log \rho_{\delta}(0) \rightarrow \rho_{0} \log \rho_{0} \text { as }\{\delta, \varepsilon\} \rightarrow 0, & \text { in } L^{1}\left(\Omega_{0}\right),\end{cases}
$$

where

$$
\nabla \bar{F}=\frac{\partial \bar{w}}{\partial t}-(\mu+\xi) \Delta \bar{w}-\xi \nabla \operatorname{div} \bar{w} \in L^{2}\left(0, T ; H^{-1 / 2}\left(\Omega_{t}\right)\right) .
$$


The elliptical regularization of the momentum equation allows us to work in an open $Q$ of $\mathbb{R}^{4}$ and to circumvent the difficulties due to the free boundary. Moreover, we will demonstrate that the regularization of the continuity equation justifies Eq. (2.13). Indeed (2.13) has been written formally and we will see that this regularisation permits to construct solutions verifying (2.13). The term $\nabla \bar{F}$ does not introduce difficulties and we can set $\nabla F=0$ without loss of generality.

Remark 2.2. - To pass to the limit, we must consider a hierarchical procedure. Indeed, we first pass to the limit on $\varepsilon$ which allows us to obtain the problem $\left(\mathcal{F}_{\delta}^{\prime}\right)$ which only depends on $\delta$. We then show that the solution of this problem satisfies the estimate (2.24) independently of $\delta$ which allows us to pass to the limit.

We now prove that the solutions of the problem $\left(\mathcal{F}_{\delta \varepsilon}^{\prime}\right)$ satisfy the estimates (2.9) and (2.24).

(c) Weak formulation and a priori estimates. We introduce $X$, a space defined by $\left\{\phi \in L^{2}(0, T ; W) ; \partial \phi / \partial t \in L^{2}(Q)\right\}$ where $W$ is given by $\left\{\phi \in H^{1}\left(\Omega_{t}\right) ; \phi . n=0\right.$ on $\left.\Gamma\right\}$ endowed of the norm of $H^{1}\left(\Omega_{t}\right)$. We endow $X$ of the norm

$$
\|\phi\|_{X}=\|\phi\|_{L^{2}(0, T ; W)}+\left\|\frac{\partial \phi}{\partial t}\right\|_{L^{2}(Q)} .
$$

We set

$$
a\left(w_{\varepsilon}, \phi\right)=\int_{Q} \operatorname{div} w_{\varepsilon} \operatorname{div} \phi+\int_{Q} \operatorname{curl} w_{\varepsilon} \operatorname{curl} \phi,
$$

and we search, for a fixed value of $\varepsilon>0$, a solution $w_{\varepsilon} \in X$ satisfying

$$
\begin{aligned}
& \varepsilon \int_{Q} \frac{\partial w_{\varepsilon}}{\partial t} \frac{\partial \phi}{\partial t}+\int_{Q} \frac{\partial w_{\varepsilon}}{\partial t} \phi+(\mu+\xi) a\left(w_{\varepsilon}, \phi\right)+\xi \int_{Q} \operatorname{div} w_{\varepsilon}, \\
& \operatorname{div} \phi=\int_{Q} \rho_{\delta} \operatorname{div} \phi, \quad \forall \phi \in X,
\end{aligned}
$$

with $w_{\varepsilon}(0) \rightarrow w_{0}$ in $L^{2}\left(\Omega_{0}\right)$ and

$$
\begin{cases}\frac{\partial \rho_{\delta}}{\partial t}+\operatorname{div}\left(\rho_{\delta} w_{\varepsilon}\right)+\operatorname{div}\left(\rho_{\delta} \bar{w}\right)+\delta \rho_{\delta}^{2}=0, & \\ \rho_{\delta} \geqslant 0, & \text { in } L^{1}\left(0, T ; W^{-1,1}\left(\Omega_{t}\right)\right), \\ \rho_{\delta}(0) \rightarrow \rho_{0}, & \text { in } L^{1}\left(\Omega_{0}\right), \\ \rho_{\delta}(0) \log \rho_{\delta}(0) \rightarrow \rho_{0} \log \rho_{0}, & \text { in } L^{1}\left(\Omega_{0}\right) .\end{cases}
$$


If $\left(w_{\varepsilon}, \rho_{\delta}\right)$ is a solution to this problem, then as $\varepsilon \rightarrow 0$

$w_{\varepsilon}$ is bounded in $L^{2}(0, T ; W)$,

$\rho_{\delta}$ is bounded in $L^{\infty}\left(0, T ; L^{1}\left(\Omega_{t}\right)\right) \cap L^{2}(Q)$,

$$
\begin{gathered}
\frac{\partial w_{\varepsilon}}{\partial t} \text { is bounded in } L^{2}\left(0, T ; W^{\prime}\right), \\
\sqrt{\varepsilon} \frac{\partial w_{\varepsilon}}{\partial t} \text { is bounded in } L^{2}(Q) .
\end{gathered}
$$

Indeed, we have

$$
\begin{gathered}
\varepsilon\left\|\frac{\partial w_{\varepsilon}}{\partial t}\right\|_{L^{2}(Q)}^{2}+\frac{1}{2}\left\|w_{\varepsilon}\right\|_{L^{\infty}\left(0, T ; L^{2}\left(\Omega_{t}\right)\right)}^{2}+(\mu+\xi)\left\|w_{\varepsilon}\right\|_{L^{2}(0, T ; W)}^{2} \\
+\xi\left\|\operatorname{div} w_{\varepsilon}\right\|_{L^{2}(Q)}^{2} \leqslant \mathbb{C}_{0}+\int_{Q} \rho_{\delta} \operatorname{div} w_{\varepsilon}-\frac{1}{2} \int_{Q_{p}} w_{\varepsilon}^{2} \frac{\partial u}{\partial t}
\end{gathered}
$$

however

$$
\frac{1}{2} \int_{Q_{p}} w_{\varepsilon}^{2} \frac{\partial g}{\partial t} \leqslant \mathbb{C}_{1}\left\|\frac{\partial u}{\partial t}\right\|_{L^{\infty}\left(0, T ; L^{2}\left(\Omega_{p}\right)\right)}\left\|w_{\varepsilon}\right\|_{L^{2}(0, T ; W)}^{2},
$$

and

$$
\begin{aligned}
\int_{Q} \rho_{\delta} \operatorname{div} w_{\varepsilon}= & -\int_{\Omega_{t}} \rho_{\delta}(t) \log \rho_{\delta}(t)+\int_{\Omega_{0}} \rho_{\delta}(0) \log \rho_{\delta}(0) \\
& -\delta \int_{Q} \rho_{\delta}^{2} \log \rho_{\delta}-\int_{Q} \rho_{\delta} \operatorname{div} \bar{w} \\
\leqslant & \mathbb{C}_{3}-\int_{\Omega_{t}} \rho_{\delta}(t) \log \rho_{\delta}(t)-\delta \int_{Q} \rho_{\delta}^{2} \log \rho_{\delta} \\
& +\left\|\rho_{\delta}\right\|_{L^{1}(Q)}\|\operatorname{div} \bar{w}\|_{L^{\infty}(Q) .}
\end{aligned}
$$

Finally, as $\rho$ is bounded in $L^{1}(Q)$ and $\operatorname{div} \bar{w}$ in $L^{\infty}(Q)$, we obtain

$$
\begin{aligned}
\varepsilon \| & \frac{\partial w_{\varepsilon}}{\partial t}\left\|_{L^{2}(Q)}^{2}+\frac{1}{2} g\right\| w_{\varepsilon} \|_{L^{\infty}\left(0, T ; L^{2}\left(\Omega_{t}\right)\right)}^{2} \\
& +\left((\mu+\xi)-\mathbb{C}_{1}\left\|\frac{\partial u}{\partial t}\right\|_{L^{\infty}\left(0, T ; L^{2}\left(\Omega_{p}\right)\right)}\right)\left\|w_{\varepsilon}\right\|_{L^{2}(0, T ; W)}^{2}
\end{aligned}
$$




$$
+\xi\left\|\operatorname{div} w_{\varepsilon}\right\|_{L^{2}(Q)}^{2}+\int_{\Omega_{t}} \rho_{\delta}(t) \log \rho_{\delta}(t)+\delta \int_{Q} \rho_{\delta}^{2} \log \rho_{\delta} \leqslant \mathbb{C}_{4},
$$

and the result follows if $\mu$ and $\xi$ are chosen sufficiently large.

(d) Passage to the limit on $\varepsilon$ and $\delta$. The above estimates permit to pass to the limit on $\varepsilon$. In particular, we have $\varepsilon\left(\partial^{2} w_{\varepsilon} / \partial t^{2}\right) \rightarrow 0$ in $\mathcal{D}^{\prime}(Q)$ and

$$
\frac{\partial w}{\partial t}-(\mu+\xi) \Delta w-\xi \nabla \operatorname{div} w+\nabla \rho_{\delta}=0 .
$$

We have obtained a problem $\left(\mathcal{F}_{\delta}^{\prime}\right)$ independent of $\varepsilon$. We must now to pass to the limit as $\delta$ goes to zero.

The $L^{2}$-regularization of $\rho_{\delta}$ is crucial since a difficulty arises in the construction of solutions satisfying (2.13). We introduce a mollifier $\left(\wp_{\varepsilon}\right)_{\varepsilon \geqslant 1}$ such that $\wp_{\varepsilon} \in C_{0}^{\infty}\left(\mathbb{R}^{3}\right)$, Supp $\wp_{\varepsilon} \subset B(0,1 / \varepsilon), \int \wp_{\varepsilon}=1$ and $\wp_{\varepsilon} \geqslant 0$. We set $\phi_{\varepsilon}=\phi * \wp_{\varepsilon}$, thus we obtain

$$
\frac{\partial \rho_{\delta \varepsilon}}{\partial t}+\operatorname{div}\left(\rho_{\delta \varepsilon} v\right)+\delta\left(\rho_{\delta}^{p^{\prime}}\right)_{\varepsilon}=r_{\varepsilon}
$$

where $r_{\varepsilon} \rightarrow 0$ ([6] Chapter 2, Lemma 2.3) in $L_{\text {loc }}^{p}(Q)$ with $1 / p=1 / 2+$ $1 / p^{\prime}$ and $p^{\prime}=2$. We set $\beta_{\mu} \in C^{1}([0, \infty))$ such that $\beta_{\mu}^{\prime}(t)=1 / \sqrt{\mu+t}$ with $0 \leqslant \mu<\infty$, we find

$$
\begin{aligned}
& \frac{\partial \beta_{\mu}\left(\rho_{\delta \varepsilon}\right)}{\partial t}+\operatorname{div}\left(\beta_{\mu}\left(\rho_{\delta \varepsilon}\right) v\right)+\operatorname{div}(v)\left(\beta_{\mu}^{\prime}\left(\rho_{\delta \varepsilon}\right) \rho_{\delta \varepsilon}-\beta_{\mu}\left(\rho_{\delta \varepsilon}\right)\right) \\
& \quad+\delta\left(\rho_{\delta}^{2}\right)_{\varepsilon} \beta_{\mu}^{\prime}\left(\rho_{\delta \varepsilon}\right)=r_{\varepsilon} \beta_{\mu}^{\prime}\left(\rho_{\delta \varepsilon}\right),
\end{aligned}
$$

but $\rho_{\delta} \in L^{2}(Q)$, therefore we can pass to the limit [8] with respect to $\varepsilon$ in (2.29), therefore

$$
\frac{\partial \beta_{\mu}\left(\rho_{\delta}\right)}{\partial t}+\operatorname{div}\left(\beta_{\mu}\left(\rho_{\delta}\right) v\right)+\operatorname{div}(v)\left(\beta_{\mu}^{\prime}\left(\rho_{\delta}\right) \rho_{\delta}-\beta_{\mu}\left(\rho_{\delta}\right)\right)+\delta \rho_{\delta}^{2} \beta_{\mu}^{\prime}\left(\rho_{\delta}\right)=0 .
$$

Finally, when $\mu$ goes to $0^{+}$in (2.30), we obtain

$$
\frac{\partial \sqrt{\rho_{\delta}}}{\partial t}+\operatorname{div}\left(\sqrt{\rho_{\delta}} v\right)-\frac{1}{2} \operatorname{div}(v) \sqrt{\rho_{\delta}}+\frac{\delta}{2} \rho_{\delta}^{3 / 2}=0 .
$$

We emphasize that in the three-dimensional situation, the $L^{2}$-regularization of the continuity equation is justified by the passage to the limit on $\varepsilon$ [8]. Indeed the estimate on $\rho$ in $L^{3 / 2}(Q)$ is insufficient. Therefore, 
we obtain a regularized equation (2.13) in which we can obtain the same estimate as in (2.24) for $\rho_{\delta}$ independently of $\delta$. We have just an additional term to estimate

$$
\begin{aligned}
\frac{\delta}{2} \int_{Q} \rho_{\delta}^{3 / 2} h & \leqslant \frac{\delta}{2}\left\|\rho_{\delta}\right\|_{L^{2}(Q)}^{3 / 2}\|h\|_{L^{4}(Q)} \leqslant \frac{3 \delta^{4 / 3}}{8}\left\|\rho_{\delta}\right\|_{L^{2}(Q)}^{2}+\frac{1}{4}\|h\|_{L^{4}(Q)}^{4} \\
& \leqslant \mathbb{C}+\frac{3 \delta^{4 / 3}}{8}\left\|\rho_{\delta}\right\|_{L^{2}(Q)}^{2} .
\end{aligned}
$$

Finally, since

$$
\int_{\Omega_{t}} \rho_{\delta}(t)+\delta\left\|\rho_{\delta}\right\|_{L^{2}(Q)}^{2}=\int_{\Omega_{0}} \rho_{\delta 0} \leqslant \mathbb{C},
$$

we find

$$
\begin{gathered}
(1-3 \varepsilon)\left\|\rho_{\delta}\right\|_{L^{3 / 2}(Q)}^{3 / 2}+\delta\left(1-\frac{3 \delta^{1 / 3}}{8}\right)\left\|\rho_{\delta}\right\|_{L^{2}(Q)}^{2} \\
\leqslant \mathbb{C}\left(1+\|w\|_{L_{t}^{12 / 5}\left(L_{x}^{3}\right)}^{3 / 2}+\|\operatorname{div} \bar{w}\|_{L_{t}^{3 / 2}\left(L_{x}^{2}\right)}^{3 / 2}\right)
\end{gathered}
$$

thus

$$
\left\|\rho_{\delta}\right\|_{L^{3 / 2}(Q)} \leqslant \mathcal{K}
$$

where $\mathcal{K}$ is an independent constant of $\delta$ and

$$
\rho_{\delta} \text { is bounded in } L^{3 / 2}(Q) \text {. }
$$

Consequently, $(\delta / 2) \rho_{\delta}^{3 / 2} \longrightarrow 0$ in $\mathcal{D}^{\prime}(Q)$ as $\delta$ goes to $0^{+}$and

$$
\frac{\partial \sqrt{\rho}}{\partial t}+\operatorname{div}(\sqrt{\rho} v)-\frac{1}{2} \operatorname{div}(v) \sqrt{\rho}=0 .
$$

Below, we use a fixed point method to construct a solution of the regularized problem.

(e) Construction of approached solutions. In this paragraph, to simplify the presentation, we suppress the indexes $\varepsilon$ and $\delta$. We note $\left\{w_{1}, \ldots, w_{i}, \ldots\right\}$, a basis of $X=\left\{\phi \in H^{1}(Q), \phi . n=0\right\}$ verifying $w_{i} \in$ $H^{5}(Q)$. We define $X_{n}$, the set of the combinations of the first $n$ functions of this basis. We introduce the following approached problem: find $w_{n}=$ 
$\sum_{j=1}^{n} a_{i} w_{i}(x, t) \in X_{n}$ and $\rho_{n} \in C^{1}(\bar{Q})$ verifying

$$
\left(\mathcal{F}_{1}^{\prime}\right)\left\{\begin{array}{cl}
\varepsilon \int_{Q} \frac{\partial w_{n}}{\partial t} \frac{\partial \phi}{\partial t}+\int_{Q} \frac{\partial w_{n}}{\partial t} \phi+(\mu+\xi) a\left(w_{n}, \phi\right) & \\
\quad+\xi \int_{Q} \operatorname{div} w_{n}, \quad \operatorname{div} \phi=\int_{Q} \rho_{n} \operatorname{div} \phi, & \forall \phi \in X_{n}, \\
w_{n}(t=0)=v_{0 n}-\bar{w}_{0} \rightarrow w_{0}, & \text { in } L^{2}\left(\Omega_{0}\right),
\end{array}\right.
$$

and

$$
\left(\mathcal{F}_{2}^{\prime}\right) \begin{cases}\frac{\partial \rho_{n}}{\partial t}+\operatorname{div}\left(w_{n} \rho_{n}\right)+\operatorname{div}\left(\bar{w}_{n} \rho_{n}\right)+\delta \rho_{n}^{2}=0, & \text { in } C^{0}(\bar{Q}) \\ \rho_{n}(t=0)=\rho_{0 n}(x)>0 \in C_{c}^{1}\left(\bar{\Omega}_{0}\right), & \\ \rho_{0 n} \rightarrow \rho_{0}>0, & \text { in } L^{1}\left(\Omega_{0}\right) \\ \rho_{0 n} \log \rho_{0 n} \rightarrow \rho_{0} \log \rho_{0} & \text { in } L^{1}\left(\Omega_{0}\right) \\ \bar{w}_{n} \rightarrow \bar{w}, & \text { in } C^{2}(\bar{Q})\end{cases}
$$

This problem is solved by using a fixed point method and we show that it admits a solution such that $\left(w_{n}, \rho_{n}\right) \in X_{n} \times C^{1}(\bar{Q})$ and $\rho_{n} \geqslant 0$.

We begin by setting $w_{n}=w_{n}^{*}$ in $\left(\mathcal{F}_{2}^{\prime}\right)$ where $w_{n}^{*}$ is a given function in $X_{n}$. The characteristics method is well adapted to solve $\left(\mathcal{F}_{2}^{\prime}\right)$. Indeed, to obtain an estimate on $\rho_{n} \log \rho_{n}$, the continuity equation must be exactly verified. We then introduce $T_{1}$, the map which at $w_{n}^{*} \in X_{n}$ associates $\rho_{n} \in L^{\infty}\left(0, T ; L^{2}\left(\Omega_{t}\right)\right)$ the solution of the problem $\left(\mathcal{F}^{\prime}\right)$ and the map $T_{2}$, which at $\rho_{n} \in L^{\infty}\left(0, T ; L^{2}\left(\Omega_{t}\right)\right)$ associates $w_{n} \in X_{n}$ a solution of the problem $\left(\mathcal{F}^{\prime}{ }_{1}\right)$ which is solved with the Galerkin method. We then define the map $T=T_{2} \circ T_{1}$ and we will show that $T$ satisfies the necessary hypothesis to apply Kakutani's fixed point theorem.

We solve $\left(\mathcal{F}^{\prime}{ }_{2}\right)$ by using the characteristics method in the following way. The curves $t \mapsto(X(x, t), t)$ are termed the characteristics of origin $x$. There is two types of characteristics, those originating from a point $x$ in $\Omega_{0}$ and, since we take the plate motions into account, those originating from a point of the boundary and which remain on the boundary. If we set $\rho_{0 n}>0$, then $\rho_{n}>0 \quad \forall t$ and we have

$$
\frac{\mathrm{d} \log \rho_{n}}{\mathrm{~d} t}+\operatorname{div} v_{n}+\delta \rho_{n}=0,
$$


moreover, by using the decomposition $w_{n}=w_{1 n}+w_{2 n}=\nabla h_{n}+\operatorname{Curl} q_{n}$, we obtain

$$
\rho_{n}=\varepsilon \frac{\partial^{2} h_{n}}{\partial t^{2}}-\frac{\partial h_{n}}{\partial t}+(\mu+2 \xi) \operatorname{div} w_{1 n}+\alpha_{\varepsilon}(t),
$$

where

$$
\alpha_{\varepsilon}(t)=\int_{\Omega_{0}} \rho_{0 n}-\varepsilon \int_{\Omega_{p}} \frac{\partial h_{n}}{\partial t} \frac{\partial u_{n}}{\partial t} \in L^{1}(0, T) .
$$

Therefore

$$
\begin{aligned}
\frac{\mathrm{d}}{\mathrm{d} t}\left\{\log \rho_{n}+\varepsilon \delta \frac{\partial h_{n}}{\partial t}-\delta h_{n}\right\}= & -\operatorname{div}\left(w_{1 n}+\bar{w}\right)+\delta(\mu+2 \xi) \operatorname{div} w_{1 n} \\
& -\delta \alpha_{\varepsilon}(t)+\delta v_{n}\left(\varepsilon \frac{\partial w_{1 n}}{\partial t}-w_{1 n}\right),
\end{aligned}
$$

and by noting $\mathbb{K}(t)$ the right hand side term, we find by integrating on the characteristics curves $\gamma_{s}$

$$
\rho_{n}(X(x, t), t)=\rho_{0 n} \exp \left(\delta\left[h_{n}-h_{0 n}\right]-\varepsilon \delta \frac{\partial h_{n}}{\partial t}+\int_{0}^{t} \mathbb{K}(t)\right) .
$$

Furthermore, the continuity equation gives us

$$
\frac{\mathrm{d}}{\mathrm{d} t}\left\|\rho_{n}\right\|_{L^{2}\left(\Omega_{t}\right)}^{2}+\delta\left\|\rho_{n}\right\|_{L^{3}(Q)}^{3}+\int_{0}^{T}\left(\operatorname{div} w_{n}^{*}+\operatorname{div} \bar{w}_{n}\right)\left|\rho_{n}\right|^{2}=0,
$$

and if the functions of the basis used to solve the problems $(\mathcal{R})$ and $\left(\mathcal{F}^{\prime}{ }_{1}\right)$ are sufficiently smooth, we obtain $\operatorname{div} w_{n_{-}}^{*}+\operatorname{div} \bar{w}_{n} \in C^{1}(\bar{Q}) \subset L^{\infty}(Q)$. Therefore, by using [8] we find $\rho_{n} \in C^{1}(\bar{Q})$ and by applying Gronwall's lemma we have

$$
\left\|\rho_{n}\right\|_{L^{\infty}\left(0, T ; L^{2}\left(\Omega_{t}\right)\right)}^{2} \leqslant\left\|\rho_{0 n}\right\|_{L^{2}\left(\Omega_{0}\right)}^{2} \exp \int_{0}^{T}\left\|\operatorname{div}\left(w_{n}^{*}+\bar{w}_{n}\right)\right\|_{L^{\infty}\left(\Omega_{t}\right)} .
$$

In the same way, we have for the momentum equation $\varepsilon\left\|\frac{\partial w_{n}}{\partial t}\right\|_{L^{2}(Q)}^{2}+\frac{1}{2}\left\|w_{n}\right\|_{L^{\infty}\left(0, T ; L^{2}\left(\Omega_{t}\right)\right)}^{2}+(\mu+2 \xi)\left\|w_{n}\right\|_{L^{2}(0, T ; W)}^{2}$ 


$$
+\xi\left\|\operatorname{div} w_{n}\right\|_{L^{2}(Q)}^{2}=\frac{1}{2}\left\|w_{0} n\right\|_{L^{2}\left(\Omega_{0}\right)}^{2}+\int_{Q} \rho_{n} \operatorname{div} w_{n}-\frac{1}{2} \int_{Q_{p}}\left|w_{n}\right|^{2} \frac{\partial g}{\partial t},
$$

and if $\partial g / \partial t$ satisfies the assumption (2.10), we obtain the following estimate

$$
\left\|w_{n}\right\|_{X_{n}}^{2} \leqslant \mathbb{C}_{1} T^{2}\left\|\rho_{n}\right\|_{L^{\infty}\left(0, T ; L^{2}\left(\Omega_{t}\right)\right)}^{2} .
$$

Finally, the inequalities (2.34) and (2.35) lead us to

$$
\left\|w_{n}\right\|_{X_{n}}^{2} \leqslant \mathbb{C}_{2} T^{2} \exp \left\|w_{n}^{*}\right\|_{X_{n}}+\mathbb{C}_{3}
$$

where $\mathbb{C}_{2}$ and $\mathbb{C}_{3}$ are two positive constants which depend exclusively on the data. Then we can apply Kakutani's theorem for which we refer to [1]. Thus, the problem $w_{n}=T\left(w_{n}^{*}\right)$ possesses a solution in $X_{n}$. The existence is obtained for a small $T$, but is true for each value of $T$ thanks to the a priori estimates.

Remark 2.3. - Because of the regularization of the momentum equation, it is natural to suppose that the stress applied to the plate by the fluid is the trace of

$$
\rho_{n}-(\mu+2 \xi) \operatorname{div}\left(w_{1 n}+\bar{w}\right)+\varepsilon \frac{\partial^{2} h_{n}}{\partial t^{2}}
$$

with $\operatorname{div} \bar{w} \in L^{\infty}\left(0, T ; H^{3 / 2}\left(\Omega_{t}\right)\right)$ and

$$
\nabla\left(\rho_{n}-(\mu+2 \xi) \operatorname{div} w_{1 n}+\varepsilon \frac{\partial^{2} h_{n}}{\partial t^{2}}\right)=-\frac{\partial w_{1 n}}{\partial t} \in L^{2}(Q),
$$

consequently

$\sigma=\sigma_{a}+\sigma_{b}=\gamma\left(\rho_{n}-(\mu+2 \xi) \operatorname{div} w_{1 n}+\varepsilon \frac{\partial^{2} h_{n}}{\partial t^{2}}\right)+\gamma(-(\mu+2 \xi) \operatorname{div} \bar{w})$

has a meaning in $L^{2}\left(0, T ; H^{1 / 2}\left(\Omega_{p}\right)\right)+L^{\infty}\left(0, T ; L^{2}\left(\Omega_{p}\right)\right)$. 


\section{2 - Resolution of the plate equation}

We can now solve the problem associated to the plate:

$$
\begin{cases}\frac{\partial^{2} u}{\partial t^{2}}-\alpha \Delta \frac{\partial^{2} u}{\partial t^{2}}+\Delta^{2} u_{\varepsilon}=f-\sigma_{a}-\sigma_{b} \in L^{2}\left(Q_{p}\right), & \text { in } Q_{p}, \\ u=\nabla u . n_{p}=0, & \text { on } \partial Q_{p}, \\ u(t=0)=u_{0}(x), & \text { in } H_{0}^{2}\left(\Omega_{p}\right), \\ \frac{\partial u}{\partial t}(t=0)=u_{1}(x), & \text { in } H_{0}^{1}\left(\Omega_{p}\right),\end{cases}
$$

with this aim in view, we use the Galerkin method with a basis of $H_{0}^{m}\left(\Omega_{p}\right)$ where $m$ is large enough. The resolution does not present any difficulties, in particular we show that $\Delta^{2} u$ and $\Delta \frac{\partial^{2} u}{\partial t^{2}}$ are bounded in $L^{2}\left(Q_{p}\right)$. Consequently, $\Delta u$ is bounded in $H^{2}\left(Q_{p}\right)$ which permits to obtain a bound on $\cos \left(\omega_{3}\right)$ in $H^{2}\left(Q_{p}\right)$. So, there exists a constant $\mathbb{K}$ such that

$$
\left\|\frac{\partial u}{\partial t} \cos \left(\omega_{3}\right)\right\|_{H^{1}\left(0, T ; H^{1}\left(\Omega_{p}\right)\right)}^{2} \leqslant \mathbb{K}\left\|\frac{\partial u}{\partial t}\right\|_{H^{1}\left(0, T ; H^{1}\left(\Omega_{p}\right)\right)}^{2} .
$$

We must verify that the solution obtained satisfies the coupling condition. To this end, we multiply the plate equation by $\partial^{2} u / \partial t^{2}$, then we integrate on $Q_{p}$. Using the estimate on $u$, we find

$$
\left\|\nabla \frac{\partial^{2} u}{\partial t^{2}}\right\|_{\left.L^{2}\left(Q_{p}\right)\right)}^{2} \leqslant \mathbb{C}\left(1+\left\|\sigma_{a}\right\|_{L^{2}\left(Q_{p}\right)}^{2}+\left\|\sigma_{b}\right\|_{L^{2}\left(Q_{p}\right)}^{2}\right)
$$

in which $\sigma_{a}$ is bounded in $L^{2}\left(Q_{p}\right)$ and $\sigma_{b}=\Delta \bar{h}$, where $\bar{h}$ is the solution to the problem $(\mathcal{R})$. Therefore $\sigma_{b}$ satisfies

$$
\left\|\sigma_{b}\right\|_{L^{2}\left(Q_{p}\right)}^{2} \leqslant \mathbb{C} T\|\operatorname{div} \bar{w}\|_{L^{\infty}\left(0, T ; H^{\frac{1}{2}}\left(\Omega_{t}\right)\right)}^{2} \leqslant \mathbb{C} T\left(1+\left\|\frac{\partial g}{\partial t}\right\|_{H^{1}\left(0, T ; H^{1}\left(\Omega_{p}\right)\right)}^{2}\right)
$$

and thus

$$
\begin{aligned}
\left\|\frac{\partial u}{\partial t} \cos \left(\omega_{3}\right)\right\|_{H^{1}\left(0, T ; H^{1}\left(\Omega_{p}\right)\right)}^{2} & \leqslant \mathbb{K}\left\|\nabla \frac{\partial^{2} u}{\partial t^{2}}\right\|_{\left.L^{2}\left(Q_{p}\right)\right)}^{2} \\
& \leqslant \mathbb{C}_{1}+\mathbb{C}_{2} T\left\|\frac{\partial g}{\partial t}\right\|_{H^{1}\left(0, T ; H^{1}\left(\Omega_{p}\right)\right)}^{2} .
\end{aligned}
$$


This last estimate permits to define the map $\Pi$ introduced above in step 4:

$$
\Pi: \frac{\partial g}{\partial t} \in H^{1}\left(0, T ; H^{1}\left(\Omega_{p}\right)\right) \mapsto \frac{\partial u}{\partial t} \cos \left(\omega_{3}\right) \in H^{1}\left(0, T ; H^{1}\left(\Omega_{p}\right)\right) .
$$

We show [1] that for all $T$ small enough, this map verifies the necessary conditions to apply Kakutani's fixed point theorem. Therefore, there exists a fixed point such that the coupling condition is checked, namely $\frac{\partial u}{\partial t} \cos \left(\omega_{3}\right)=\frac{\partial g}{\partial t}=v \cdot n_{b}$ in $H^{1}\left(0, T ; H^{1}\left(\Omega_{p}\right)\right)$. We note that, thanks to the a priori estimates, this result can be extended for all $T$, which completes the proof.

\section{REFERENCES}

[1] Chatelon F.J., Orenga P., On a non-homogeneous shallow water problem, Mathematical Modelling and Numerical Analysis 31 (1) (1997) 27-55.

[2] Flori F., Orenga P., On a fluid-structure interaction problem in velocity displacement formulation, Mathematical Models and Methods in Applied Sciences 8 (4) (1998) 543-572.

[3] Flori F., Orenga P., Analysis of a nonlinear fluid-structure interaction problem in velocity displacement formulation, Nonlinear Analysis 35 (5) (1999) 561-587.

[4] Flori F., Orenga P., On a nonlinear fluid-structure interaction problem defined on a domain depending on time, Nonlinear Analysis 38 (5) (1999) 549-569.

[5] Flori F., Orenga P., On a fluid-structure interaction problem, in: Trends in Applications of Mathematics to Mechanics, Monographs and Surveys in Pure and Applied Mathematics, Vol. 106, Chapman \& Hall/CRC, 2000, pp. 293-305.

[6] Lions P.L., Mathematical Topics in Fluid Mechanics, Vols.1 \& 2, Oxford University Press, 1998.

[7] Orenga P., Un théorème d'existence de solution d'un problème de shallow water, Archive Rational Mech. Anal. 130 (1995) 183-204.

[8] di Perna R.J., Lions P.L., Ordinary differential equations, transport theory and Sobolev spaces, Invent. Math. 98 (3) (1989) 511-547. 